\title{
Direct Observation of Photoinduced Bound Charge-Pair States at an Organic-Inorganic Semiconductor Interface
}

\author{
Yana Vaynzof, Artem A. Bakulin, ${ }^{*}$ Simon Gélinas, and Richard H. Friend ${ }^{\dagger}$ \\ Cavendish laboratory, University of Cambridge, J J Thomson Avenue, Cambridge CB3 OHE, United Kingdom
}

(Received 12 December 2011; published 15 June 2012)

\begin{abstract}
It is generally considered that photoinduced charge transfer at the organic-inorganic interfaces in hybrid photovoltaic devices immediately results in a pair of free charge carriers. We extend a novel interfaceselective ultrafast "optical pump-push photocurrent probe" technique to study hybrid photovoltaic systems and observe bound electron-hole pair states at the organic-inorganic interface formed between electron-accepting zinc oxide and electron-donating conjugated polymers. We estimate that $\sim 50 \%$ of photogenerated charges stay bound and later recombine, thus hindering the photovoltaic performance of polymer $/ \mathrm{ZnO}$ cells. We further demonstrate that interface modification with a fullerene derivative decreases the fraction of bound charges to $\sim 25 \%$, which substantially improves the device efficiency.
\end{abstract}

DOI: 10.1103/PhysRevLett.108.246605

PACS numbers: 72.40.+w, 73.61.Ph, 78.47.J-

Organic, hybrid, and dye-sensitized photovoltaic devices (PVs) are an emerging technology with the potential for an easy-to-process, low-cost, and environmentally friendly renewable energy source [1-5]. The active layer in such devices typically consists of an organic donor and an organic or inorganic acceptor materials. A photon absorbed by the donor material produces a singlet exciton which, upon reaching the donor-acceptor interface, can efficiently dissociate into a pair of charges. It recently became generally accepted that, due to the low dielectric constant of organic materials, the photogenerated charge carriers are not efficiently screened from each other and their electrostatic attraction can limit the PV efficiency. For all-organic PVs, the phenomenon of such a bound charge-transfer (CT) state forming at the donor-acceptor interface was recently identified and comprehensively studied [6-10]. Only in certain material systems, like polymer-fullerene blends, have recent experiments shown efficient and fieldindependent charge separation indicating that the bound CT states are less significant for such PV devices [11].

The higher dielectric constant of inorganic materials, however, is considered to promote efficient decoupling of charge carriers due to the increased screening of electronhole interaction. This idea inspired extensive research and development of hybrid organic-inorganic devices $[2,3,12,13]$ where, upon illumination, excitons are created in the highly absorbing organic material and efficiently dissociated at the interface with the inorganic counterpart of the device. However, these promising hybrid photovoltaic devices show very moderate performance $[14,15]$. One possible reason for this could be the existence of bound charge pairs $(\mathrm{BCP})$ at the organic-inorganic interfaces. Here, we define $\mathrm{BCP}$ as a state comprising of a positive charge localized on an organic molecule (polaron) Coulombically attracted to an electron in the inorganic material. These states may serve as a major loss mechanism limiting the hybrid solar cell performance, similarly to what has been previously demonstrated for CT states in all-organic PVs. Despite indirect indications that such a scenario can take place [16-22] the lack of an interface sensitive time-resolved technique to selectively probe $\mathrm{BCP}$ states at organic-inorganic interfaces has complicated the elucidation the physical processes governing the charge separation in hybrid photovoltaics.

Here we extend a newly developed method to selectively study the yield and dynamics of BCP states localized at the organic-inorganic semiconductor interface and apply it to a set of "model" hybrid bi-layer PV devices. We demonstrate that these states exist at the interface between a compact layer of $\mathrm{ZnO}$ and two different conjugated polymers. We show that the amount of BCP states can be controlled by interfacial modification, drastically improving the device performance.

Figure 1(a) presents the schematic structure of the hybrid photovoltaic devices examined in this study. The device active layer consisted of a single $\sim 200 \mathrm{~nm}$ layer of poly(3-hexylthiophene) (P3HT) or poly( (9,9-dioctylfluorene)-2,7-diyl-alt-[4,7-bis(3-hexylthien-5-yl)2,1,3-benzothiadiazole]-2',2"'-diyl) (F8TBT) polymers deposited on top of a $40 \mathrm{~nm}$ thick film of $\mathrm{ZnO}$. To test the (a)

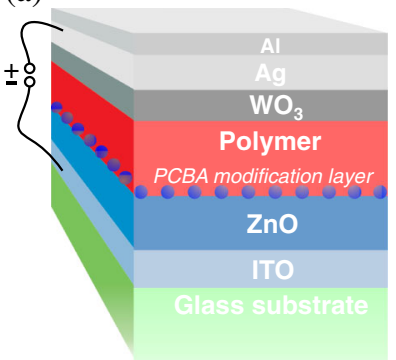

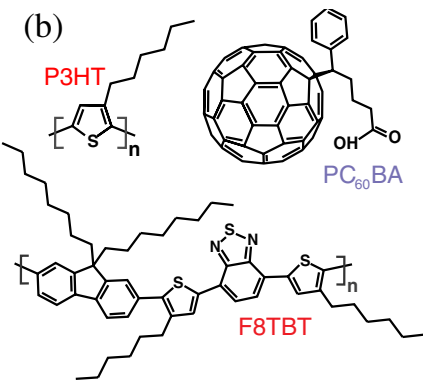

FIG. 1 (color online). (a) Structure of hybrid bi-layered photovoltaic devices. (b) Chemical structure of organic compounds used in the study. 
effect of organic-inorganic interface modification, similar devices with a monolayer of phenyl-C61-butyric acid (PCBA) between the polymer and oxide layers were fabricated. The PCBA modification, in this case, has been shown to increase the substrate work function due to an interfacial dipole [23]. The chemical structures of the materials are shown in Fig. 1(b).

Figure 2 shows the external quantum efficiencies (EQE) of the polymer/ZnO and polymer/PCBA/ZnO photovoltaic devices. Unmodified photovoltaic devices of F8TBT/ZnO and $\mathrm{P} 3 \mathrm{HT} / \mathrm{ZnO}$ demonstrate a very modest peak EQE of $2 \%$ and $3.5 \%$, respectively. This low efficiency might be caused by several different reasons. First, only a small fraction of incident light is absorbed within the exciton diffusion length from the polymer/ZnO interface, creating excitons that can be harvested for a photocurrent. Second, electron-hole pairs at the polymer/ZnO interface can form BCP states and undergo geminate recombination rather than separate into free charge carriers. Finally, poor charge transport and extraction by the electrodes may hinder the device performance.

Interestingly, photovoltaic devices with PCBA interfacial modification demonstrate substantially higher peak $\mathrm{EQE}$ values of 5\% and $10 \%$. We have previously suggested that the relatively high $\mathrm{EQE}$ value of $10 \%$ for this simple bilayer structure results from a very efficient charge separation at the interface [22]. We note that despite the clear increase in EQE upon PCBA modification, the performance of the F8TBT devices is inferior to that of P3HT. This is a result of poor charge transport in the F8TBT polymer in comparison to P3HT, as confirmed by the increased F8TBT EQE measured for thinner photovoltaic devices (not shown here) and, possibly, a smaller exciton diffusion range.

To elucidate the dynamics of charge separation at the organic-inorganic interface and to investigate the origin of the EQE increase upon interfacial modification, we applied the newly developed [24] ultrafast pump-push photocurrent technique and extended this method to perform a quantitative analysis of charge generation yields [Fig. 3(a)]. The

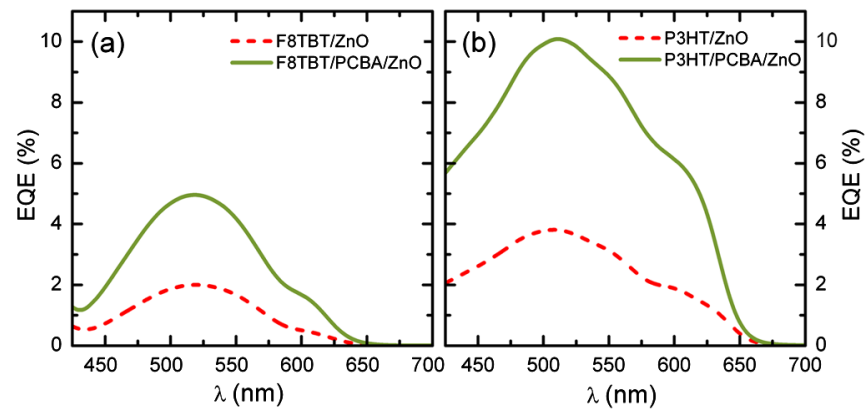

FIG. 2 (color online). External quantum efficiency (EQE) spectra of investigated devices based on (a) F8TBT and (b) P3HT. For EQE measurements, a $250 \mathrm{~W}$ tungsten halogen lamp and an Oriel Cornerstone 130 monochromater were used. The measurements were performed as a function of wavelength at intensities $\sim 1 \mathrm{~mW} / \mathrm{cm}^{2}$. technique combines previous efforts in visible and UV pump-push photocurrent experiments [25] with a gentle and targeted control over photoconversion using lowenergy IR photons [24]. Moreover, in the current study, the pump-push technique is applied to photovoltaic cells with a well-controlled single heterojunction which allows a comparison of the absolute concentration of bound states in different devices. As shown below, this quantitative information, together with a global analysis of the results, provides information about the exact yield of bound and free charges in the system.

In the experiment, the photovoltaic cell is first exposed to a $40 \mathrm{pJ}$ visible-light $(580 \mathrm{~nm}) 200 \mathrm{fs}$ pump pulse from a noncolinear optical parametric amplifier, mimicking sunlight excitation; this triggers the light-to-current conversion process. Holes, created in the polymer after exciton dissociation, alter the electronic structure of the polymer chains due to lattice relaxation, creating self-localized positive polarons which absorb photons with an energy $\sim 0.3-0.6 \mathrm{eV}$ [26,27]. After a certain delay time, a $0.4 \mu \mathrm{J}, 2200 \mathrm{~nm}(0.56 \mathrm{eV}), 250 \mathrm{fs}$ IR push pulse from another optical parametric amplifier (TOPAS, Light Conversion) illuminates the PV cell. This pulse is selectively absorbed by the hole polarons, as the neutral polymer chains are transparent in this spectral region. Although the concentration of polarons generated at the organicinorganic interface is low (less than one charge pair per $\left.1600 \mathrm{~nm}^{2}\right)$ the high flux of IR photons used is required to bring some $(\sim 0.1 \%)$ of polarons to the "hot" excited state [24]. In this way, the IR push photons provide the all hole

(a)
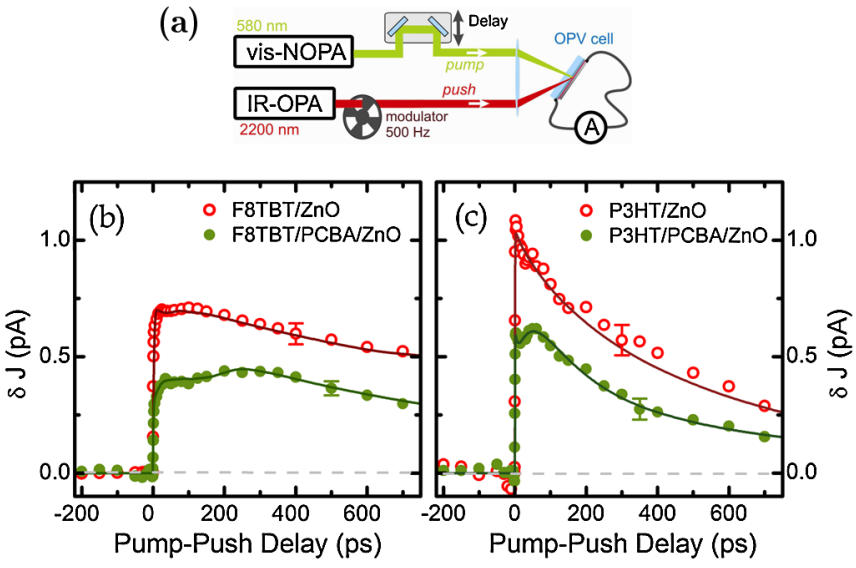

FIG. 3 (color online). (a) The layout of pump-push photocurrent spectroscopy setup. NOPA and OPA stand for (noncollinear) optical parametric amplifier. (A) stands for a lock-in amplifier in the current mode phase locked to the mechanical modulator. The energy of the pump pulse was $40 \mathrm{pJ}$ (into a $\sim 0.2 \mathrm{~mm}$ diameter spot) and the energy of the IR push $0.4 \mu \mathrm{J}$ (into a $\sim 0.5 \mathrm{~mm}$ diameter spot). Results of pump-push photocurrent $(\delta J)$ measurements on (b) F8TBT and (c) P3HT hybrid devices. Measurements were performed in identical experimental conditions. Solid lines are fits to the data with the model presented in the Fig. 4. The error bars reflect the typical standard error for the corresponding transients. 
polarons with "excess" energy, allowing them to reach otherwise energetically inaccessible configurations. Therefore, the positive polarons in the BCP states can be released from the electron in the $\mathrm{ZnO}$ and converted to free charge carriers thus contributing to the photocurrent. Alternatively, the push can excite the electron in $\mathrm{ZnO}$ and relocalize it away from the interface; however, this latter scenario is unlikely because charges in $\mathrm{ZnO}$ absorb in the different spectral region $(0.1-0.3 \mathrm{eV})$ [28]. In the experiment, we detect the effect of the push pulse by monitoring the increase of the photocurrent output $(\delta J)$ of the photovoltaic device. As the PCBA-modified and unmodified devices were measured under the same conditions, the absolute value $\delta J$ can be used to deduce the change in device performance upon modification. We note that free positive polarons contribute to the photocurrent without the push pulse and do not affect the experimental observable $\delta J$, making the newly developed technique selective solely to bound states.

Figures 3(b) and 3(c) show the change in the photocurrent due to the push as a function of the pump-push delay for the F8TBT and P3HT hybrid devices, respectively. When the push pulse arrives before the pump, the effect on the photocurrent is negligible since there are very few charges in the cell to be influenced by the IR photons. When the push arrives after the pump, the $\delta J$ manifestly increases for all the PV devices. This is direct evidence of bound positive polarons present on the polymer. The sharp increase in the $\delta J$ signal is dominated by a prompt component (within the system $\sim 250 \mathrm{fs}$ time resolution) demonstrating that the majority of bound polarons are generated on an ultrafast time scale. Since exciton dissociation occurs exclusively at the organic-inorganic interface and bound polarons are observed on this ultrafast time scale, we conclude that they are of interfacial character and belong to BCP states. This is the first selective observation of BCP states formed across organic-inorganic interfaces.

Our observation of BCP states at a hybrid interface is analogous to the reported observation of CT states in allorganic photovoltaics [6,7]. However, the nature of $\mathrm{BCP}$ states is not identical to that of CT states. Both comprise a positive polaron on the organic polymer chain adjacent to the interface, but the electron wave function is very different. For the zinc oxide, it will be derived from a delocalized band state, and polaronic relaxation is not expected to be significant. Local defects at the zinc oxide surface may, however, assist the localization of the electron state.

This pump-push optical technique allows us to monitor the generation and recombination dynamics of $\mathrm{BCP}$ states (Fig. 3). As mentioned above, the majority of BCP states are generated instantly; however, an additional component is observed to grow up to around 200 ps. We consider that this component, particularly pronounced for PCBA-modified cells, is due to the delayed arrival of singlet excitons to the interface. The later decay of $\delta J$ is associated with the geminate recombination of BCP states. Although bi-molecular effects (like bound-free charge recombination) may potentially affect the dynamics of BCP states, the low pump fluxes used to reproduce "sun-illumination" conditions eliminate the possibility of substantial nongeminate contributions to the dynamics. This was confirmed by a linear dependence of the photocurrent on the pump intensity and no change of the shape of the $d J(t)$ curve with increased intensity of the pump pulse.

The employment of this pump-push technique in conjunction with photovoltaic characterization gives us an opportunity to investigate the consequences of the BCP states' formation on the PV performance. We observe that the initial increase in photocurrent due to the push pulse is only $\sim 30 \%$ lower for PCBA-modified devices. This is qualitatively consistent with the observed threefold increase in EQE when modified by PCBA [23]. To analyze quantitatively the yield of $\mathrm{BCP}$ states and their effect on the photovoltaic performance, we assume that the push pulse has a similar effect on BCP states in PCBA-modified and unmodified devices. Then, a large increase in the amount of free charges (measured in EQE) caused by only a moderate decrease in the amount of bound charges is an indicator of a high yield of BCP states at the unmodified interface.

In addition to an increase in charge-separation efficiency, we find that additional processes of exciton harvesting are present in PCBA-modified devices. The evidence for this phenomenon is clearly visible in Figs. 3(b) and 3(c)-while the transients for polymer/ZnO devices decay nearly monotonically, those measured on the PCBA-modified devices show an additional growth component with the time constant of $\sim 150 \mathrm{ps}$ (F8TBT) or $\sim 60$ ps (P3HT). There are several possible contributions to the enhanced light harvesting originating from the PCBA modification. First, altering the surface energy of the substrate may cause morphological changes in the polymer packing at the interface $[13,29]$. This in turn can create a favorable energetic landscape for exciton migration to the interface. Alternatively, one can associate enhanced harvesting with a long-range Forster energy transfer from the polymer to the PCBA monolayer. Recent studies by Liu et al. illustrate this possibility, as they indicate that the range of "multidipole" energy transfer may substantially exceed the exciton diffusion length [30]. To conclude, the favorable effect of PCBA on device performance is twofold; PCBA monolayer reduces the amount of BCP states and causes an increase in the amount of excitons reaching the interface.

To determine the yield of BCP states and to disentangle the different contributions to the EQE improvement, we developed a state model, presented in Fig. 4(a), with the corresponding band diagrams in Figs. 4(b) and 4(c). According to this model, charges are generated upon exciton diffusion $(A)$ and, additionally, in the case of PCBA-modified devices, upon enhanced long-range exciton harvesting, as discussed above $(B)$. The fractional contribution of the latter process is given by $\boldsymbol{\omega}=$ $\boldsymbol{B} /(\boldsymbol{A}+\boldsymbol{B})$. Generated charge pairs can either remain as BCP states (and subsequently geminately recombine), with fraction $\gamma$, or dissociate into free charge carriers to 
(a)

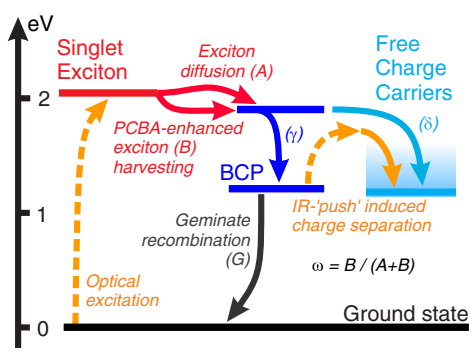

(b)

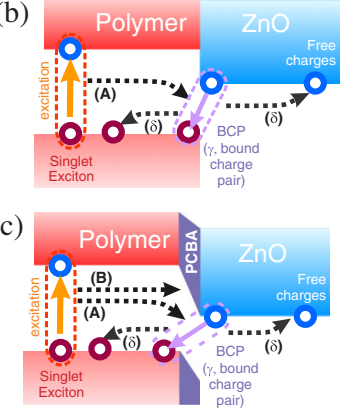

FIG. 4 (color online). (a) The state model of charge separation. Dashed arrows represent optical transitions driven by pump and push pulses. $A$ and $B$ are two channels of exciton transfer to the organic-inorganic interface. $A$ is exciton diffusion and $B$ is additional exciton harvesting promoted by the PCBA modification of the interface. $\boldsymbol{\gamma}$ and $\boldsymbol{\delta}$ represent the relative amount of BCP and free charges, respectively. (b), (c) Band diagram of photoconversion in hybrid photovoltaic devices. Dashed arrows illustrate the processes described by the model in (a). The parameters of the fit with the model are shown in Table I.

contribute to the photocurrent, with fraction $\boldsymbol{\delta}=\mathbf{1}-\boldsymbol{\gamma}$. At longer time scales (250-700 ps) the recombination kinetics $(G)$ are similar for both the polymer/ZnO and the polymer/PCBA/ZnO devices, suggesting that no new states are created in the polymer and, therefore, justifying the assumption that both $(A)$ and $(B)$ share the same prefactor $\boldsymbol{\gamma}$. The value of $\boldsymbol{\delta}$ depends on the presence of a PCBA monolayer at the interface. The proposed model can be described by the kinetic equation:

$$
\frac{d[B C P(t)]}{d t}=\gamma[A(t)+B(t)]-G(t)[\mathrm{BCP}(t)],
$$

where $A(t), B(t)$, and $G(t)$ are the (time-varying) generation rates and recombination rates of $\mathrm{BCP}$ states, respectively. The model was then incorporated in a global fitting procedure to determine the kinetics of $A(t), B(t)$, and $G(t)$. Because of the complex nature of these processes, we chose to treat the particularities of charge dynamics phenomenologically and avoid relying on ungrounded assumptions about the physical processes underlying the observed dynamics. As optimizing this problem involves a large parameter space with many local minima, we used a genetic algorithm instead of more traditional gradient-based methods.

In analogy with biological evolution, the algorithm starts with a large ensemble of randomly generated solutions and evaluates the fitness of each of them (i.e., the inverse of the residual between the solution and the data set) [31]. Solutions of high fitness are more likely to survive and reproduce, leading to a new generation of "children" composed of linear combinations of high-fitness parents. A small amount of mutations is randomly added to preserve the diversity in the solution ensemble. As successive generations are bred, the solution converges to an optimum (see Supplemental Material for details [32]). The data sets presented in Figs. 3(b) and 3(c) were fitted with this procedure and the results are presented as solid lines. The

global parameters obtained by the optimization procedure were then used in a quantitative interpretation of the obtained results.

Knowing the EQEs as well as relative amplitudes of $A(t)$ and $B(t)$ measured for both with- and without-PCBA devices $\left(A_{\text {no_PCBA }}, B_{\text {no_PCBA }}=0, A_{\text {with_PCBA }}, B_{\text {with_PCBA }}\right)$, we obtain a quantitative measure of the absolute yields of all processes involved in photoconversion by solving the following equations:

$$
\begin{gathered}
A \times \gamma_{\mathrm{no} \_ \text {PCBA }}=A_{\text {no_PCBA }}, \quad A \times \gamma_{\text {with_PCBA }}=A_{\text {with_PCBA }}, \\
B \times \gamma_{\text {with_PCBA }}=B_{\text {with_PCBA }}, \\
\frac{(A+0)\left(1-\gamma_{\text {no_PCBA }}\right)}{(A+B)\left(1-\gamma_{\text {with_PCBA }}\right)}=\frac{E Q E_{\text {no_PCBA }}}{E Q E_{\text {with_PCBA }}} .
\end{gathered}
$$

The resulting parameters $(\boldsymbol{\delta}, \boldsymbol{\gamma}$ and $\boldsymbol{\omega})$ are summarized in Table I while further details of the fitting are presented in the Supplemental Material [32].

In the case of the unmodified $\mathrm{P} 3 \mathrm{HT} / \mathrm{ZnO}$ devices, we find that $\gamma=52 \%$ of photoinduced charges remain in BCP states. Similarly, in the case of the F8TBT/ZnO devices, the amount of BCP states $(\gamma)$ is calculated to be $54 \%$. This demonstrates that over half of the photoinduced charges remain in BCP states at the unmodified organic-inorganic interface, making it a major loss mechanism in hybrid photovoltaics.

The PCBA modification approximately halves the yield of BCP states for both polymers. Additionally, the $35 \%$ (P3HT) or $45 \%$ (F8TBT) enhanced exciton harvesting contributes to the increased photovoltaic performance. Together both contributions result in a $300 \%$ improvement in efficiency, which is consistent with the EQE measurements. Therefore, the beneficial effect of the PCBA is twofold: improved exciton harvesting and decreased number of BCP states.

It is remarkable that the percentage of BCP states determined from the genetic-algorithm modeling is so high. This indicates that the effect of the high dielectric constant of the oxide is not facilitating the BCP state dissociation process. We speculate that the nature of BCP states is related to the existence of defect states on the surface of the $\mathrm{ZnO}$ [33]. Upon exciton dissociation at the polymer-oxide interface, the electron might be localized in a surface defect state, Coulombically attracting the hole polaron on the polymer

TABLE I. Parameters of the fit [Fig. 3(b) and 3(c)] with the model presented in Fig. 4. $\boldsymbol{\gamma}$ and $\boldsymbol{\delta}$ are the relative amounts of $\mathrm{BCP}$ and free charges formed after exciton dissociation. $\boldsymbol{\omega}$ is the fractional contribution of PCBA-enhanced light harvesting to charge generation. Error values reflect the spread over different samples.

\begin{tabular}{lccc}
\hline \hline & $\boldsymbol{\gamma}$ & $\boldsymbol{\delta}$ & $\boldsymbol{\omega}$ \\
\hline F8TBT/ZnO & $54 \pm 5 \%$ & $46 \pm 5 \%$ & $\cdots$ \\
F8TBT/PCBA/ZnO & $24 \pm 3 \%$ & $76 \pm 3 \%$ & $35 \pm 10 \%$ \\
P3HT/ZnO & $52 \pm 5 \%$ & $48 \pm 5 \%$ & $\cdots$ \\
P3HT/PCBA/ZnO & $28 \pm 3 \%$ & $72 \pm 3 \%$ & $45 \pm 10 \%$ \\
\hline \hline
\end{tabular}


chain, resulting in a BCP state formation. Arguably, a similar effect could hinder the efficiency of dye-sensitized solar cells [34]. In these cells, back recombination of electron-hole pairs across the hybrid interface is a major loss mechanism [35]. This suggests the existence of localized electrons at the surface of the inorganic layer resulting in the formation of BCP states. Modifying the interface with a PCBA monolayer changes not only the energy level alignment at the interface with $\mathrm{ZnO}$, but also increases electronhole separation, promoting BCP dissociation.

In conclusion, we report the first direct observation of the presence of bound charge-pair states and demonstrate their detrimental effect on the charge-separation dynamics at the organic-inorganic interface. The yield of such states is unexpectedly high, $\sim 55 \%$, despite the high dielectric constant of the inorganic material, which is believed to reduce Coulombic attraction between the electron and hole at the interface. We show that the efficiency of exciton harvesting and disassociation to free charge carriers strongly depends on the properties of the interface, and can be substantially enhanced by an organic monolayer modification of the inorganic surface. The current results are of fundamental importance for an understanding of the photo-physical processes taking place in organic-inorganic hybrid photovoltaic cells.

We thank A. Rao, N. Greenham, and D. Kabra for useful discussions. A. A. B. acknowledges the Netherlands Organization for Scientific Research (NWO) for support through Rubicon and VENI grants. This project was supported by the Engineering and Physical Sciences Research Council. Y. V. and A. A. B. contributed equally to the manuscript.

*Present address: FOM Institute AMOLF, Science Park 104, 1098 XG, Amsterdam, The Netherlands.

†Corresponding author rhf10@cam.ac.uk

[1] K. M. Coakley and M.D. McGehee, Chem. Mater. 16, 4533 (2004).

[2] W. U. Huynh, J. J. Dittmer, and A. P. Alivisatos, Science 295, 2425 (2002).

[3] M. Law, L. E. Greene, J. C. Johnson, R. Saykally, and P. Yang, Nature Mater. 4, 455 (2005).

[4] R.-Q. Png, P.-J. Chia, J.-C. Tang, B. Liu, S. Sivaramakrishnan, M. Zhou, S.-H. Khong, H.S.O. Chan, J. H. Burroughes, L.-L. Chua, R. H. Friend, and P. K. H. Ho, Nature Mater. 9, 152 (2009).

[5] G. Dennler, M. C. Scharber, and C. J. Brabec, Adv. Mater. 21, 1323 (2009).

[6] T. M. Clarke and J. R. Durrant, Chem. Rev. 110, 6736 (2010).

[7] C. Deibel, T. Strobel, and V. Dyakonov, Adv. Mater. 22, 4097 (2010).

[8] A. A. Bakulin, S. A. Zapunidy, M.S. Pshenichnikov, P. H. M. v. Loosdrecht, and D. Y. Paraschuk, Phys. Chem. Chem. Phys. 11, 7324 (2009).

[9] D. Veldman, S. C. J. Meskers, and R. A. J. Janssen, Adv. Funct. Mater. 19, 1939 (2009).
[10] K. Vandewal, K. Tvingstedt, A. Gadisa, O. Inganas, and J. V. Manca, Nature Mater. 8, 904 (2009).

[11] M. C. Scharber, C. Lungenschmied, H.-J. Egelhaaf, G. Matt, M. Bednorz, T. Fromherz, J. Gao, D. Jarzab, and M. A. Loi, Energy Environ. Sci. 4, 5077 (2011).

[12] B. O'Regan and M. Gratzel, Nature (London) 353, 737 (1991).

[13] A. L. Briseno, T.W. Holcombe, A. I. Boukai, E. C. Garnett, S. W. Shelton, J. J. M. Frechet, and P. Yang, Nano Lett. 10, 334 (2010).

[14] D. C. Olson, J. Piris, R. T. Collins, S. E. Shaheen, and D. S. Ginley, Thin Solid Films 496, 26 (2006).

[15] P. Ravirajan, A. M. Peiro, M. K. Nazeeruddin, M. Graetzel, D. D. C. Bradley, J.R. Durrant, and J. Nelson, J. Phys. Chem. B 110, 7635 (2006).

[16] I. Haeldermans, K. Vandewal, W. D. Oosterbaan, A. Gadisa, J. D. Haen, M. K. V. Bael, J. V. Manca, and J. Mullens, Appl. Phys. Lett. 93, 223302 (2008).

[17] F. Witt, M. Kruszynska, H. Borchert, and J. Parisi, J. Phys. Chem. Lett. 1, 2999 (2010).

[18] H. Nemec, J. Rochford, O. Taratula, E. Galoppini, P. Kuzel, T. Polivka, A. Yartsev, and V. Sundstrom, Phys. Rev. Lett. 104, 197401 (2010).

[19] P. Tiwana, P. Docampo, M. B. Johnston, H. J. Snaith, and L. M. Herz, ACS Nano 5, 5158 (2011).

[20] S. Ardo, Y. Sun, A. Staniszewski, F. N. Castellano, and G. J. Meyer, J. Am. Chem. Soc. 132, 6696 (2010).

[21] A. Y. Anderson, P. R. F. Barnes, J. R. Durrant, and B. C. O’Regan, J. Phys. Chem. C 114, 1953 (2010).

[22] D. Herrmann, S. Niesar, C. Scharsich, A. Kohler, M. Stutzmann, and E. Riedle, J. Am. Chem. Soc. 133, 18220 (2011).

[23] Y. Vaynzof, D. Kabra, L. Zhao, P. K. H. Ho, A. T.-S. Wee, and R. H. Friend, Appl. Phys. Lett. 97, 033309 (2010).

[24] A. A. Bakulin, A. Rao, V. G. Pavelyev, P. H.M.v. Loosdrecht, M.S. Pshenichnikov, D. Niedzialek, J. Cornil, D. Beljonne, and R.H. Friend, Science 335, 1340 (2012).

[25] J. G. Müller, J. M. Lupton, J. Feldmann, U. Lemmer, M. C. Scharber, N. S. Sariciftci, C. J. Brabec, and U. Scherf, Phys. Rev. B 72, 195208 (2005).

[26] C.-X. Sheng, M. Tong, S. Singh, and Z. V. Vardeny, Phys. Rev. B 75, 085206 (2007).

[27] T. Drori, J. Holt, and Z. V. Vardeny, Phys. Rev. B 82, 075207 (2010).

[28] E. V. Lavrov, F. Borrnert, and J. Weber, Phys. Rev. B 72, 085212 (2005).

[29] N. Sai, K. Leung, and J. R. Chelikowsky, Phys. Rev. B 83, 121309 (2011).

[30] Y.-X. Liu, M. A. Summers, S. R. Scully, and M.D. McGehee, J. Appl. Phys. 99, 093521 (2006).

[31] M. Srinivas and L. M. Patnaik, IEEE Transactions on Systems, Man, and Cybernetics 24, 656 (1994).

[32] See Supplemental Material at http://link.aps.org/ supplemental/10.1103/PhysRevLett.108.246605 for a detailed description of the genetic algorithm fitting procedure.

[33] L. Schmidt-Mende and J.L. MacManus-Driscoll, Mater. Today 10, 40 (2007).

[34] L. Peter, Accounts Chem. Res. 42, 1839 (2009).

[35] A. Listorti, B. O'Regan, and J. R. Durrant, Chem. Mater. 23, 3381 (2011). 